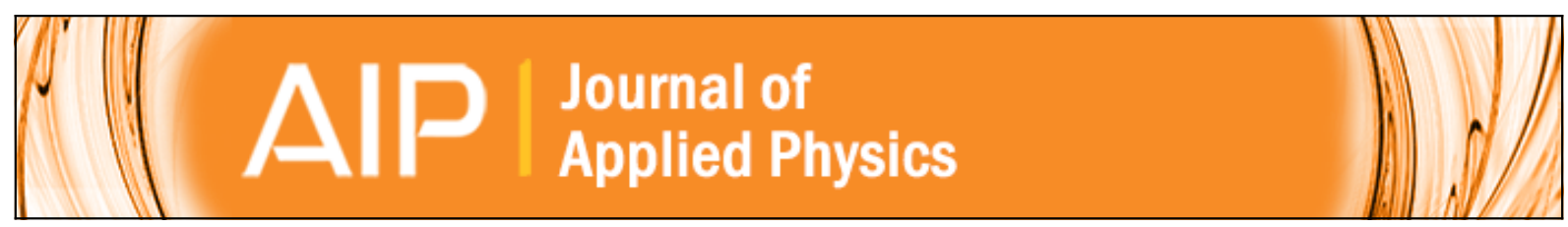

\title{
Step-edge-induced resistance anisotropy in quasi-free-standing bilayer chemical vapor deposition graphene on SiC
}

Tymoteusz Ciuk, Semih Cakmakyapan, Ekmel Ozbay, Piotr Caban, Kacper Grodecki, Aleksandra Krajewska, Iwona Pasternak, Jan Szmidt, and Wlodek Strupinski

Citation: Journal of Applied Physics 116, 123708 (2014); doi: 10.1063/1.4896581

View online: http://dx.doi.org/10.1063/1.4896581

View Table of Contents: http://scitation.aip.org/content/aip/journal/jap/116/12?ver=pdfcov

Published by the AIP Publishing

\section{Articles you may be interested in}

Preparation and electrical transport properties of quasi free standing bilayer graphene on SiC (0001) substrate by $\mathrm{H}$ intercalation

Appl. Phys. Lett. 105, 183105 (2014); 10.1063/1.4901163

Nitrogen doping of chemical vapor deposition grown graphene on $4 \mathrm{H}-\mathrm{SiC}(0001)$

J. Appl. Phys. 115, 233504 (2014); 10.1063/1.4884015

Graphene growth on AIN templates on silicon using propane-hydrogen chemical vapor deposition

Appl. Phys. Lett. 104, 071912 (2014); 10.1063/1.4866285

Detection of polar chemical vapors using epitaxial graphene grown on $\mathrm{SiC}$ (0001)

Appl. Phys. Lett. 102, 173103 (2013); 10.1063/1.4803511

The influence of substrate morphology on thickness uniformity and unintentional doping of epitaxial graphene on SiC

Appl. Phys. Lett. 100, 241607 (2012); 10.1063/1.4729556

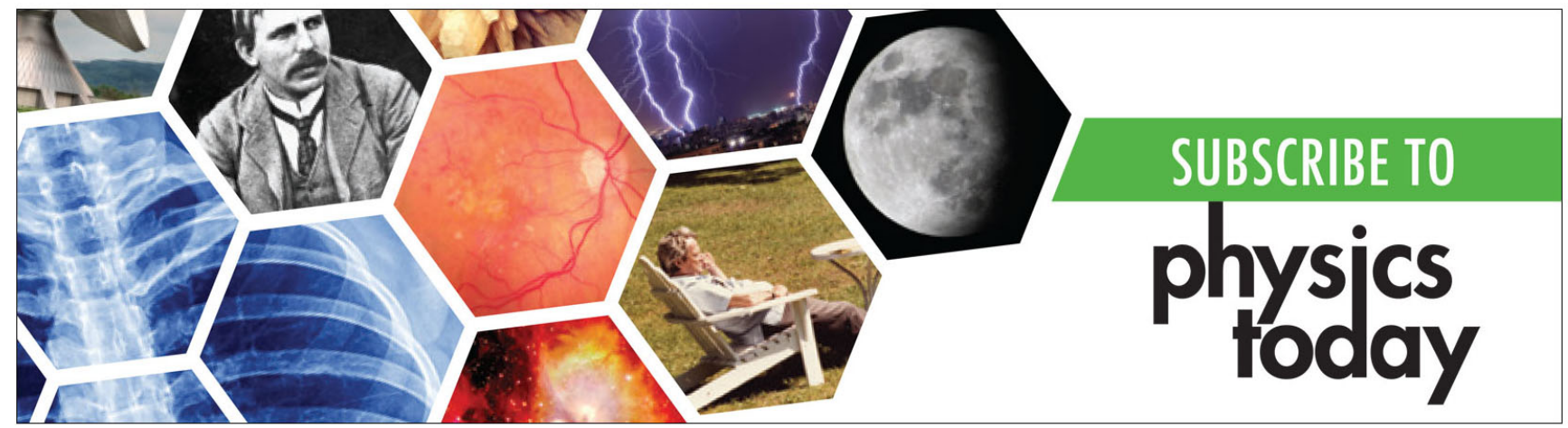




\title{
Step-edge-induced resistance anisotropy in quasi-free-standing bilayer chemical vapor deposition graphene on SiC
}

\author{
Tymoteusz Ciuk, ${ }^{1,2}$ Semih Cakmakyapan, ${ }^{3}$ Ekmel Ozbay, ${ }^{3}$ Piotr Caban, ${ }^{1}$ Kacper Grodecki, ${ }^{1}$ \\ Aleksandra Krajewska, ${ }^{1,4}$ Iwona Pasternak, ${ }^{1}$ Jan Szmidt, ${ }^{2}$ and Wlodek Strupinski ${ }^{1, a)}$ \\ ${ }^{1}$ Institute of Electronic Materials Technology, Wolczynska 133, 01-919 Warsaw, Poland \\ ${ }^{2}$ Institute of Microelectronics and Optoelectronics, Warsaw University of Technology, Koszykowa 75 , \\ 00-662 Warsaw, Poland \\ ${ }^{3}$ Department of Electrical and Electronics Engineering, Department of Physics, Nanotechnology Research \\ Center, Bilkent University, 06800 Bilkent, Ankara, Turkey \\ ${ }^{4}$ Institute of Optoelectronics, Military University of Technology, Gen. S. Kaliskiego 2, 00-908 Warsaw, Poland
}

(Received 14 June 2014; accepted 15 September 2014; published online 25 September 2014)

The transport properties of quasi-free-standing (QFS) bilayer graphene on SiC depend on a range of scattering mechanisms. Most of them are isotropic in nature. However, the SiC substrate morphology marked by a distinctive pattern of the terraces gives rise to an anisotropy in graphene's sheet resistance, which may be considered an additional scattering mechanism. At a technological level, the growth-preceding in situ etching of the SiC surface promotes step bunching which results in macro steps $\sim 10 \mathrm{~nm}$ in height. In this report, we study the qualitative and quantitative effects of $\mathrm{SiC}$ steps edges on the resistance of epitaxial graphene grown by chemical vapor deposition. We experimentally determine the value of step edge resistivity in hydrogen-intercalated QFS-bilayer graphene to be $\sim 190 \Omega \mu \mathrm{m}$ for step height $\mathrm{h}_{\mathrm{S}}=10 \mathrm{~nm}$ and provide proof that it cannot originate from mechanical deformation of graphene but is likely to arise from lowered carrier concentration in the step area. Our results are confronted with the previously reported values of the step edge resistivity in monolayer graphene over $\mathrm{SiC}$ atomic steps. In our analysis, we focus on large-scale, statistical properties to foster the scalable technology of industrial graphene for electronics and sensor applications. (C) 2014 AIP Publishing LLC. [http://dx.doi.org/10.1063/1.4896581]

\section{INTRODUCTION}

Owing to its outstanding carrier mobility, graphene has been expected to realize high-speed electronics, ${ }^{1,2}$ however the room-temperature carrier mobility of $140000 \mathrm{~cm}^{2} / \mathrm{Vs}$ was only reported for its exfoliated form on $\mathrm{BN}^{3}$, in excess of $15000 \mathrm{~cm}^{2} / \mathrm{Vs}$ on $\mathrm{SiO}_{2}{ }^{4-6}$ and $25000 \mathrm{~cm}^{2} / \mathrm{Vs}$ for its suspended form ${ }^{7}\left(200000 \mathrm{~cm}^{2} / \mathrm{Vs}\right.$ at low temperature $\left.{ }^{8,9}\right)$. When graphene is grown on $\mathrm{SiC}$ substrate, its carrier transport is significantly affected by a range of scattering mechanisms, predominantly, long-range Coulomb scattering on charged impurities trapped in the graphene-substrate interface. ${ }^{10-30}$ Others include short-range disorder related to intrinsic lattice imperfections, point defects and dislocations, ${ }^{10,11,20,22,24-27,31-34}$ "ripples" in graphene's atomic structure, ${ }^{6,7,35-38}$ and acoustic phonons. ${ }^{39-41}$ It has been calculated that in case of monolayer graphene, the room temperature intrinsic mobility of charge carriers is phononlimited to $\sim 10^{5} \mathrm{~cm}^{2} / \mathrm{Vs}$ (Refs. $39-41$ ) and the most plausible sources of scattering are charged impurities. The mean free path for short-range scatterers $l_{S}$ is proportional to $1 / \sqrt{n}$, where $n$ is the charge carrier density. For Coulomb scatterers due to the screening effect, $l_{S} \sim \sqrt{n}$, therefore, short-range scattering must be included into formalism only for very low ionized impurity density or at high carrier densities. A simple analytic equation was derived to relate the charged-

\footnotetext{
a) Author to whom correspondence should be addressed. Electronic mail: wlodek.strupinski@itme.edu.pl
}

impurity-limited mobility, $\mu \times n_{\text {imp }}=5 \times 10^{15} \mathrm{~V}^{-1} \mathrm{~s}^{-1}, 14,18$ where $n_{i m p}$ is the effective impurity concentration and it is suggested that reducing the typical $n_{\text {imp }}$ in present day samples, $n_{\text {imp }} \sim 10^{11}-10^{12} \mathrm{~cm}^{-2}$, by two orders of magnitude should increase the mobility to $\sim 10^{5} \mathrm{~cm}^{2} / \mathrm{Vs}$, giving way to the short-range scattering model. In bilayer graphene, the screening effect is quantitatively much stronger than in monolayer graphene. The resultant is the scattering mechanism of the over-screened Coulomb impurities which is equally important as the short-range disorder. ${ }^{24,25}$

In the most promising technology for wafer-scale production of graphene devices, ${ }^{2,42}$ i.e., sublimation ${ }^{43-45}$ and Chemical Vapor Deposition (CVD) growth on $\mathrm{SiC},{ }^{46}$ surface substrate morphology also acts effectively as a scattering mechanisms. The role of SiC morphology on transport properties of graphene grown by silicon sublimation was discussed by several groups. ${ }^{47-49}$ It has been reported that $\mathrm{SiC}$ step edge density, ${ }^{50}$ step height, ${ }^{51}$ and step bunching ${ }^{52,53}$ give rise to graphene's resistance. The step edge resistivity in monolayer graphene was evaluated by scanning potentiometry in a scanning tunneling microscope ${ }^{51}$ and later associated with the abrupt variation in potential and doping due to detachment of graphene from the substrate as it passes over a step. ${ }^{54}$ Both experiments were related to $\mathrm{SiC}$ atomic steps and proved step edge resistivity $\rho_{\text {step }} \approx 15 \Omega \mu \mathrm{m}$ and $\rho_{\text {step }} \approx 25 \Omega \mu \mathrm{m}$ for step height $h_{s}$ equal to $1.0 \mathrm{~nm}$ and $1.5 \mathrm{~nm}$, respectively. Consequently, the surplus resistance introduced by step edges was explained through carrier depletion rather than an additional scattering mechanism. In Ref. 55, it is 
suggested that the conduction anisotropy is a reflection of both geometric anisotropy and the extent of residual silicon atoms aggregated at the step edges, where they enhance carrier scattering.

Graphene grown on $\mathrm{Si}$ face of $\mathrm{SiC}$ rests on a buffer layer which is the first layer of carbon atoms covalently bound to the substrate. ${ }^{56-58}$ It can be decoupled to form a quasi-freestanding bilayer graphene (QFS-bilayer) through hydrogen atoms intercalation. ${ }^{59}$ The intercalating atoms diffuse underneath the buffer layer and bound themselves to the topmost $\mathrm{Si}$ atoms of the $\mathrm{SiC}$ substrate converting the buffer layer to a mostly sp2-hybridized monolayer graphene. The resultant QFS-bilayer graphene is partly screened from the substrate and exhibits on average three times higher carrier mobility than the un-intercalated one. Importantly, its transport properties are not degraded up to $700{ }^{\circ} \mathrm{C}$. Therefore, it is mostly suited for high-speed applications. Unfortunately, the growth-preceding in situ etching of the SiC surface promotes step bunching which results in macro steps $\sim 10 \mathrm{~nm}$ in height, as opposed to much lower atomic steps investigated in Refs. 51 and 54. The step bunching is expected to considerably increase the step edge resistivity. In this report, we examine the qualitative and quantitative effects of SiC steps on graphene's resistance and experimentally determine the value of $\rho_{\text {step }}$ in hydrogen-intercalated bilayer graphene.

\section{EXPERIMENTAL DETAILS}

In this paper, we studied the effect of the step-edgeinduced resistance anisotropy in hydrogen intercalated, QFSbilayer graphene on the $\mathrm{Si}$ face of $4 \mathrm{H}-\mathrm{SiC}(0001)$ and $6 \mathrm{H}-$ $\mathrm{SiC}(0001)(10 \mathrm{~mm} \times 10 \mathrm{~mm})$. The investigated samples were grown using the CVD method on semi-insulating on-axis substrates in a standard hot-wall CVD Aixtron VP508 reactor. Prior to the growth, in situ etching of the SiC surface was carried out in hydrogen atmosphere. The epitaxial CVD growth of graphene was realized under dynamic flow conditions that simultaneously inhibit $\mathrm{Si}$ sublimation and promote the mass transport of propane molecules to $\mathrm{SiC}$ substrate. ${ }^{46}$ The growth process was followed by in situ hydrogen intercalation at $1000{ }^{\circ} \mathrm{C}$ in $900 \mathrm{mbar} \mathrm{Ar}$ atmosphere. The as grown samples were characterized by Hall effect measurements in van der Pauw geometry with the four golden probes placed in the corners of the $10 \mathrm{~mm} \times 10 \mathrm{~mm}$ substrates. Altogether 140 4H-SiC and $606 \mathrm{H}-\mathrm{SiC}$ samples were fabricated and investigated to assure a statistical perspective. Typical values of hole concentration obtained at room temperature were of the order of $1.3 \times 10^{13} \mathrm{~cm}^{-2}$ and their mobility proved on average $2500 \mathrm{~cm}^{2} / \mathrm{Vs}$ (up to $5300 \mathrm{~cm}^{2} / \mathrm{Vs}$ ).

The qualitative influence of $\mathrm{SiC}$ step edges resulting from step bunching on the average resistance of QFS-bilayer graphene was in the first place derived from standard Hall effect characterization in van der Pauw geometry with the use of an 0.55T Ecopia HMS-3000 setup. Prior to the measurement, each graphene sample was inspected under an optical microscope and assigned a specific angle of the $\mathrm{SiC}$ terraces configuration. The terraces appear to follow a uniform direction over the entire area of a substrate. Fig. 1(a) illustrates the adopted convention for the angle assignment.
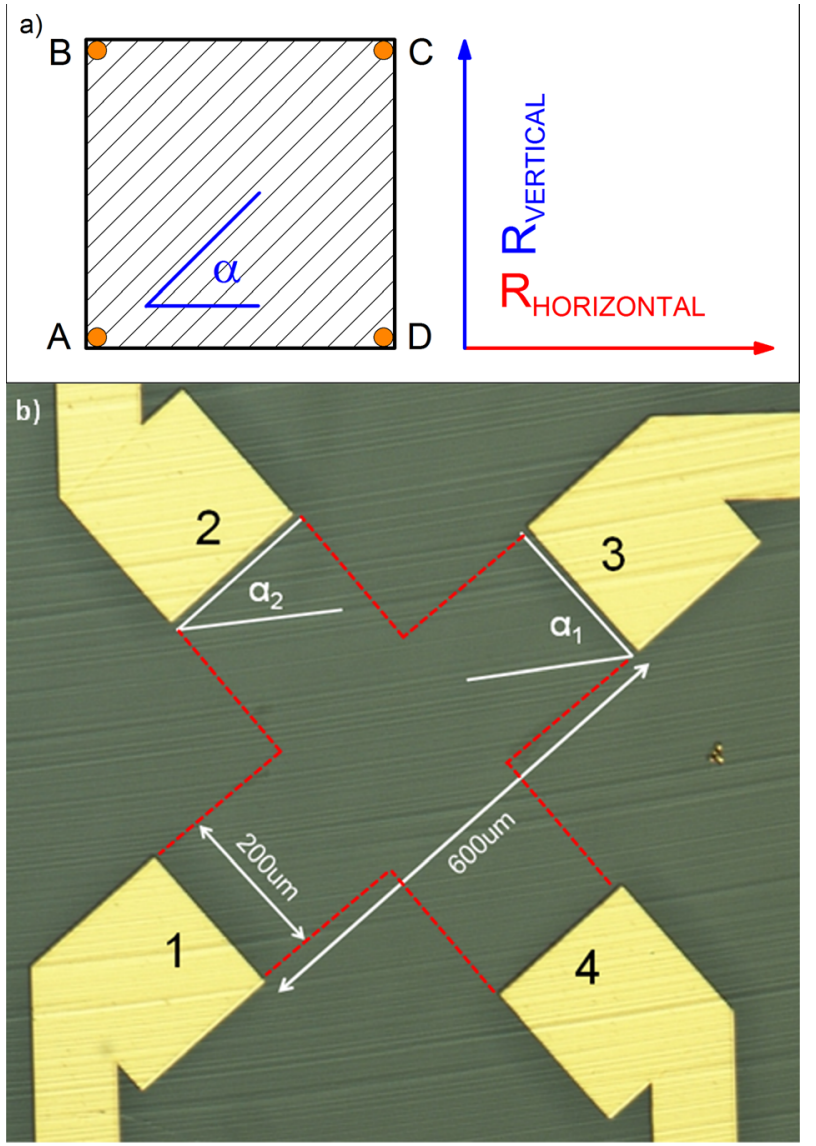

FIG. 1. (a) Schematic view of a SiC substrate $(10 \mathrm{~mm} \times 10 \mathrm{~mm})$ and the adopted convention for the angle $\alpha$ assignment of the terraces orientation with respect to the SiC substrate edges. Letters A-D indicate four corners of the substrate, where the four golden pins were placed during the standard Hall effect characterization in van der Pauw geometry. This approach was adopted to qualitatively observe the step-edge-induced resistance anisotropy in graphene. (b) Optical image of a photolithographically patterned equalarm graphene Hall cross designed for the quantitative analysis of the step edge resistivity.

The resultant is $\alpha$ ranging from $0^{\circ}$ to $90^{\circ}$ with the terraces running horizontally $\left(\alpha=0^{\circ}\right)$, vertically $\left(\alpha=90^{\circ}\right)$, or at any other angle calculated from the level.

In the standard van der Pauw method for the sheet resistance determination, it is required to measure the $R_{A B}, C D$, $R_{C D, A B}, R_{A D, B C}, R_{B C, A D}$, auxiliary resistances in the first place. These values are defined as $\left(V_{D}-V_{C}\right) / I_{A B},\left(V_{B}-V_{A}\right) /$ $I_{D C},\left(V_{C}-V_{B}\right) / I_{A D}$, and $\left(V_{D}-V_{A}\right) / I_{B C}$, respectively. Based on this, the $R_{V E R T I C A L}$ and $R_{\text {HORIZONTAL }}$ are calculated as arithmetic means of $\left(R_{A B}, C D, R_{C D}, A B\right)$ and $\left(R_{A D, B C}\right.$, $R_{B C}, A D$ ),respectively. It can be shown that the following relation holds (van der Pauw ${ }^{60}$ ):

$$
\exp \left(-\pi R_{V E R T I C A L} / R_{S}\right)+\exp \left(-\pi R_{\text {HORIZONTAL }} / R_{S}\right)=1,
$$

where $R_{S}$ is the material's sheet resistance. In this report, the $R_{V E R T I C A L}$ and $R_{\text {HORIZONTAL values are considered to be }}$ influenced by the terraces orientation and are confirmed to follow a precise function of the angle $\alpha$. Both resistances are related to a hypothetical $R_{A V E R A G E}$ that corresponds to $R_{S}$ through $2 \exp \left(-\pi R_{A V E R A G E} / R_{S}\right)=1$, where $R_{S}$ is determined by $R_{V E R T I C A L}$ and $R_{\text {HORIZONTAL }}$. Each of the 200 verified samples was subject to two subsequent measurements 
( $\mathrm{I}=1 \mathrm{~mA}$ ) with the second preceded by sample rotation by $90^{\circ}$, thus rearranging the corners from $A B C D$ to $D A B C$. With this approach, our statistical perspective was doubled. The anisotropy in transport properties is manifested in normalized values of $R_{\text {VERTICAL }} / R_{A V E R A G E}$ and $R_{\text {HORIZONTAL }} /$ $R_{A V E R A G E}$ in the function of $\alpha$.

The qualitative observation of step-edge-induced resistance anisotropy provides a justification for further quantitative analysis. Two hydrogen intercalated QFS-bilayer 4H$\mathrm{SiC}$ samples were photolithographically patterned to form nine graphene Hall bars on each. Initial van der Pauw characterization proved that both graphene samples displayed similar parameters: hole concentration $\mathrm{n} \approx 1.8 \times 10^{13} \mathrm{~cm}^{-2}$, carrier mobility $\mu \approx 2300 \mathrm{~cm}^{2} / \mathrm{Vs}$, and sheet resistance $\mathrm{R}_{\mathrm{S}} \approx 150 \Omega / \mathrm{sq}$. The nine Hall bars took the form of symmetrical, equal-arm crosses rotated at a gradually increasing angle $\left(0^{\circ}, 5^{\circ}, 10^{\circ}, 15^{\circ}, 20^{\circ}, 25^{\circ}, 30^{\circ}, 35^{\circ}, 40^{\circ}\right)$ with respect to the substrate's edges and hence to $\mathrm{SiC}$ terraces. Each of the two bars forming the graphene cross had $200 \mu \mathrm{m}$ in width and $600 \mu \mathrm{m}$ in length. $20 \mathrm{~nm} \_\mathrm{Ti} / 80 \mathrm{~nm} \_\mathrm{Au}$ ohmic contacts $(200 \mu \mathrm{m} \times 200 \mu \mathrm{m})$ were e-beam deposited. Fig. 1(b) illustrates one of the nine crosses.

In order to deepen our understanding of the origin of the step edge resistivity, an additional sample with a transferred graphene was produced. Graphene grown on $12 \mu$ m thick $3 \mathrm{~N}$ JTCHTE GOULD Electronics copper foil in Aixtron VP508 reactor was transferred onto a $4 \mathrm{H}-\mathrm{SiC}$ substrate through the PMMA-mediated electrochemical delamination method. ${ }^{61}$ Prior to transfer, the substrate underwent a process of hydrogen etching in $1600{ }^{\circ} \mathrm{C}$ to promote step bunching on its surface. An identical pattern of nine rotated Hall bars was fabricated accordingly to the above presented details.

In each of the nine Hall crosses, the specific $\alpha_{1}$ and $\alpha_{2}$ angles were determined. $\alpha_{1}$ corresponds to step edge orientation in the graphene channel between contacts " 1 " and " 3 ," $\alpha_{2}$ between " 2 " and " 4 ." The configuration of a Hall cross provides two mutually perpendicular graphene resistors $R_{13}$ and $R_{24}$. It can be judged from the photograph that the total resistance between two opposite contact pads is described by the following formulas:

$$
\begin{aligned}
& R_{13}=R_{C 1}+R_{\text {channel } 13}\left(\alpha_{1}\right)+R_{C 3}, \\
& R_{24}=R_{C 2}+R_{\text {channel } 24}\left(\alpha_{2}\right)+R_{C 4},
\end{aligned}
$$

where $R_{C 1} \ldots R_{C 4}$ are the contact resistances and $R_{\text {channel13 }}\left(\alpha_{1}\right)$ and $R_{\text {channel } 24}\left(\alpha_{2}\right)$ denote the resistance of $200 \mu \mathrm{m} \times 600 \mu \mathrm{m}$ graphene channels. It was assessed with additional TLM (Transfer Length Method) structures featuring $200 \mu \mathrm{m} \times 200 \mu \mathrm{m}$ pads located next to the equal-arm crosses that the unit length contact resistance varied between $600 \Omega \mu \mathrm{m}$ and $1100 \Omega \mu \mathrm{m}$, indicating that a single $200 \mu \mathrm{m} \times 200 \mu \mathrm{m}$ contact pad introduces between $3 \Omega$ and $5.5 \Omega$. In order to verify possible angle dependence of the contact resistance, the TLM structures were fabricated at a range of angles with respect to $\mathrm{SiC}$ terraces and multiplied for each orientation, so that the results had a statistical perspective. In the analyzed sample, these angles lied in the range between $40^{\circ}$ and $90^{\circ}$. The experimental values proved no angle dependence (Fig. 2). The authors believe that in any given cross
$R_{C 1}$ and $R_{C 3}$, as well as $R_{C 2}$ and $R_{C 4}$ are mutually approximately equal and their difference is negligible with respect to the expected value of $R_{\text {channel13 }}\left(\alpha_{1}\right)-R_{\text {channel24 }}\left(\alpha_{2}\right)$.

Based on the measured values of $R_{13}$ and $R_{24}$, one can calculate the following relation:

$$
\Delta R(\Delta \alpha)=\left|R_{13}-R_{24}\right|, \quad \text { where } \quad \Delta \alpha=\left|\alpha_{1}-\alpha_{2}\right|
$$

$\Delta R(\Delta \alpha)$ reflects the differential resistance between two perpendicular graphene channels as a function of the differential angle $\Delta \alpha$. For $\alpha_{1}=\alpha_{2}=45^{\circ}$ and $\Delta \alpha=0$, which holds for identical terrace orientation in both channels, $\Delta R(\Delta \alpha)$ is expected to account for zero. When $\Delta \alpha=90^{\circ}, \Delta R(\Delta \alpha)$ reaches its maximum value and equals the excess resistance introduced by a finite number of $\mathrm{SiC}$ step edges that are $200 \mu \mathrm{m}$ wide and cover the entire graphene channel. The authors chose to locally define a $\Delta R(\Delta \alpha)$ relation for each of the equal-arm crosses to minimize possible influence of graphene's quality inhomogeneity that if occurred throughout the sample would interfere with the influence of SiC step edges. The nine Hall crosses provide nine data points for a linear fit that reproduces the $\Delta R(\Delta \alpha)$ relation. We later use this fitted relation in the form of $y=a x+b$ to calculate the exact value of

$$
\Delta R\left(\Delta \alpha=90^{\circ}\right)=a \times 90^{\circ}=n_{\text {edges }} \times \rho_{\text {step }} / 200 \mu \mathrm{m},
$$

where $n_{\text {edges }}$ is the number of SiC step edges. The intercept $b$ is intentionally neglected as it is attributed other than terrace origin, however the authors cannot provide a meaningful explanation for its origin. The accurate number of SiC step edges $n_{\text {edges }}$ over the distance of $600 \mu \mathrm{m}$ is specific for each sample and was determined with the use of an atomic force microscope. Based on $a \times 90^{\circ}=n_{\text {edges }} \times \rho_{\text {step }} / 200 \mu \mathrm{m}$, the average resistivity $\rho_{\text {step }}[\Omega \mu \mathrm{m}]$ of a single $\mathrm{SiC}$ step edge in QFS-bilayer graphene was derived.

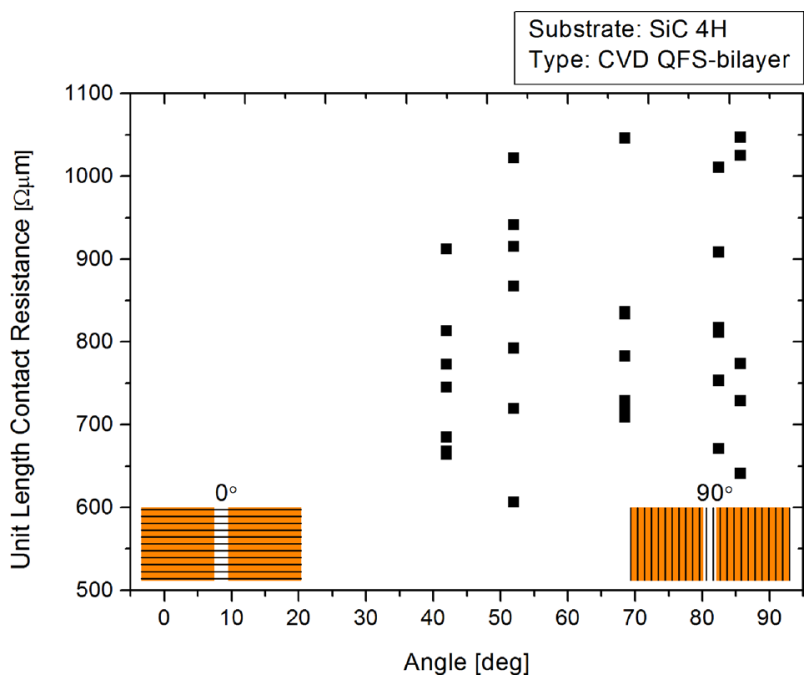

FIG. 2. Statistical analysis of the unit length contact resistance angle dependence measured in TLM structures featuring $200 \mu \mathrm{m} \times 200 \mu \mathrm{m}$ contact pads. The TLM structures were rotated at different angles with respect to $\mathrm{SiC}$ terraces and multiplied to illustrate possible data distribution for the same angle. The insets schematically illustrate the terrace orientation between two adjacent TLM pads and the adopted convention for angle assignment. 


\section{EXPERIMENTAL RESULTS}

The qualitative observation of the step-edge-induced resistance anisotropy in hydrogen intercalated graphene on $4 \mathrm{H}-$ $\mathrm{SiC}(0001)$ and $6 \mathrm{H}-\mathrm{SiC}(0001)(10 \mathrm{~mm} \times 10 \mathrm{~mm})$ substrates is presented in Figs. 3(a) and 3(b), respectively. The convention for the angle assignment of the terraces orientation with respect to the sample edges was depicted in Fig. 1(a). The $R_{\text {VERTICAL }} / R_{A V E R A G E}$ and $R_{\text {HORIZONTAL }} / R_{A V E R A G E}$ data points, where $R_{V E R T I C A L}$ and $R_{\text {HORIZONTAL }}$ are the auxiliary van der Pauw resistances and $R_{A V E R A G E}$ is a hypothetical quantity that corresponds to $R_{S}$ through $2 \exp \left(-\pi R_{A V E R A G E} / R_{S}\right)=1$, clearly illustrate the cumulative effect of $\mathrm{SiC}$ step edges on the total resistance of QFS-bilayer graphene. The lowest normalized resistance is observed in the direction parallel to the SiC terraces $\left(\alpha=0^{\circ}\right.$ for $R_{\text {HORIZONTAL }} / R_{A V E R A G E}$ and $\alpha=90^{\circ}$ for $\left.R_{V E R T I C A L} / R_{A V E R A G E}\right)$. It gradually increases as the step edges effectively hinder the current flow. In the direction perpendicular to the terraces, the normalized resistance reaches its maximum $\left(\alpha=90^{\circ}\right.$ for $R_{\text {HORIZONTAL }} / R_{A V E R A G E}$ and $\alpha=0^{\circ}$ for
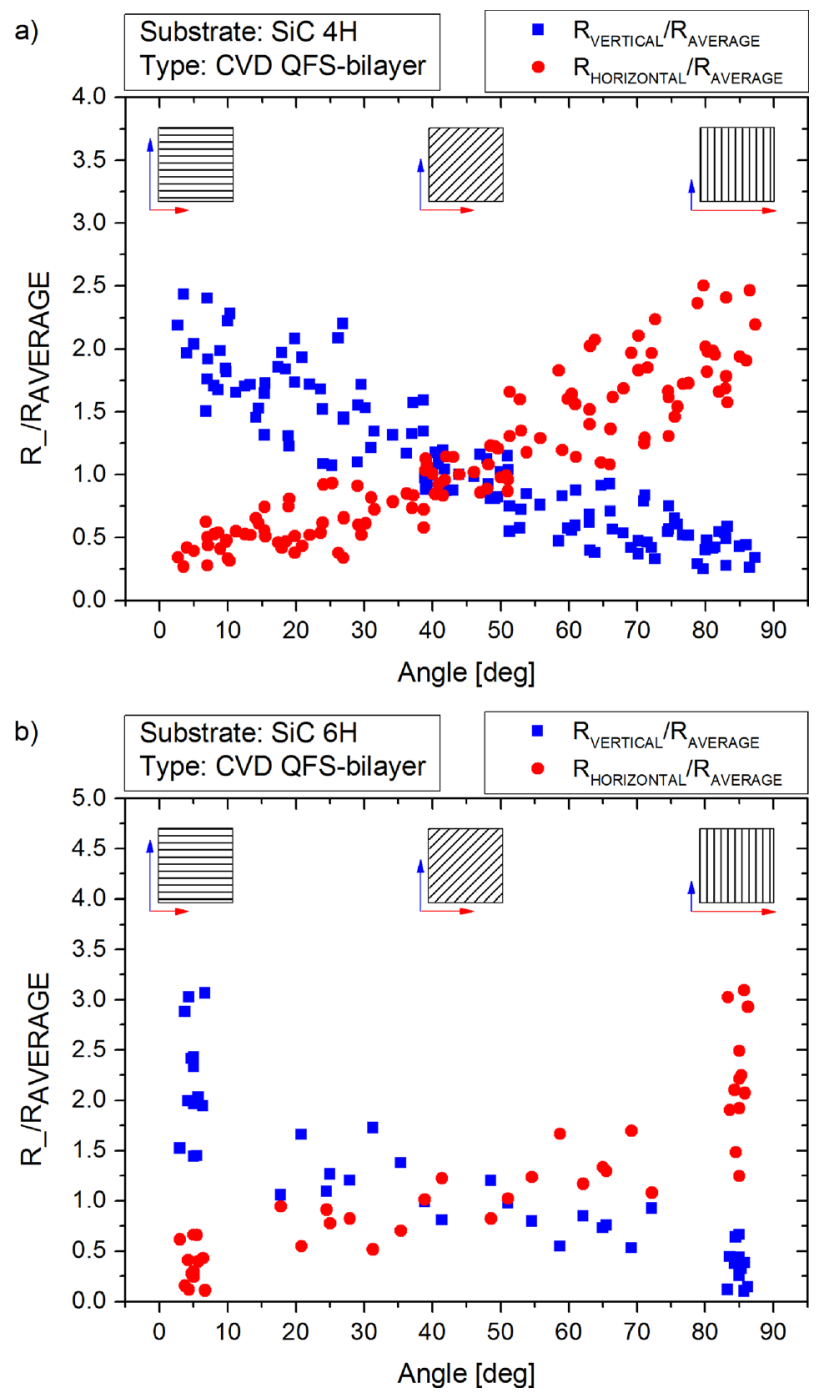

FIG. 3. The qualitative observation of the resistance anisotropy $\left(R_{V E R T I C A L}\right)$ $R_{A V E R A G E}$ and $R_{\text {HORIZONTAL }} / R_{A V E R A G E}$ in the function of the terraces orientation with respect to the sample edges in CVD QFS-bilayer graphene measured in van der Pauw geometry on the surface of $10 \mathrm{~mm} \times 10 \mathrm{~mm}$ samples. (a) $4 \mathrm{H}-\mathrm{SiC}(0001)$ and (b) $6 \mathrm{H}-\mathrm{SiC}(0001)$.
$R_{V E R T I C A L}\left(R_{A V E R A G E}\right)$. Both datasets are mutually symmetrical and cross exactly at the angle of $45^{\circ}$, which is in agreement with the expectations. Qualitatively similar results are obtained for $4 \mathrm{H}-\mathrm{SiC}$ and $6 \mathrm{H}-\mathrm{SiC}$ substrates. It happened that within the set of $606 \mathrm{H}-\mathrm{SiC}$ samples, a majority displayed terraces oriented along the substrate's edges, hence an accumulation of data points around $5^{\circ}$ and $85^{\circ}$. The significantly larger spread of resistance values at these angles as compared with intermediate steps is explained by the fact that within a more numerous set, one encounters a wider distribution of the total number of terraces, which translates into the observable data span. The statistical analysis of 200 samples proves that the step edges constitute a non-negligible mechanism of carrier transport impediment.

The quantitative description of the average step edge resistivity $\rho_{\text {step }}$ is brought by the Hall crosses rotated at a varying angle with respect to $\mathrm{SiC}$ terraces (Fig. 1(b)). The measured $\Delta R(\Delta \alpha)$ relation of the two hydrogen intercalated $4 \mathrm{H}-\mathrm{SiC}$ samples is depicted in Fig. 4. $\Delta R$ is the differential resistance between two perpendicular graphene channels and $\Delta \alpha$ is the differential angle between the terraces orientation in the two channels. It is expected that when $\alpha_{1}=\alpha_{2}=45^{\circ}$ and $\Delta \alpha=\left|\alpha_{1}-\alpha_{2}\right|=0$, which holds for identical terraces orientation in both channels, $\Delta R$ should equal 0 and reach its maximum for $\Delta \alpha=90^{\circ}$, when in one channel, the terraces run parallel to the direction of the current flow and perpendicular in the other. It was observed that for every $\alpha_{1}<\alpha_{2}$, $R_{13}>R_{24}$, and $R_{13}<R_{24}$ for $\alpha_{1}>\alpha_{2}$. This is indicative of the step-edge-induced resistance anisotropy and it is consistent with the above reported qualitative observation that the more terraces hamper the current flow the higher the resistance. The collected data points were linear fitted with $y=a x+b$ and yield the slope $a$ equal to 0.915 and 1.393 . Contrary to expectations for $\Delta \alpha=0$, the intercept $b \neq 0(\Delta R \neq 0)$. We attribute it other than terrace origin and intentionally neglect in the determination of $\rho_{\text {step }}$. Both samples were inspected

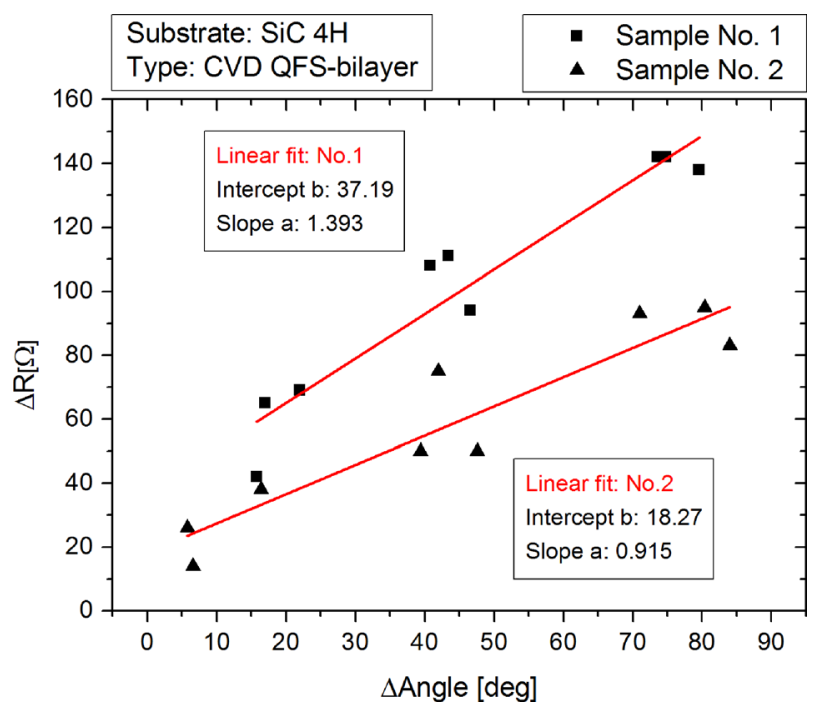

FIG. 4. Differential resistance $\Delta R$ measured in nine pairs of mutually perpendicular graphene channels $(200 \mu \mathrm{m} \times 600 \mu \mathrm{m})$ as a function of the differential angle $\Delta \alpha$ between the terraces orientation in each pair of the mutually perpendicular channels fabricated on two QFS-bilayer 4H-SiC(0001) samples. 
with a NanoScope Controller driven Veeco Dimension V atomic force microscope equipped with an OTESPA cantilever. A $2 \mathrm{~mm}$-long line scan in the direction perpendicular to the terraces was performed in order to statistically define an average number of $\mathrm{SiC}$ step edges $n_{\text {edges }}$ over the distance of $600 \mu \mathrm{m}$, which is the length of either of the graphene channels. The two samples were assigned $n_{\text {edges }} \sim 81$ and $\sim 130$, respectively. Based on Eq. (1), the resultant step edge resistivity was calculated $203 \Omega \mu \mathrm{m}$ and $192 \Omega \mu \mathrm{m}$, accordingly.

Given these, one can relate the calculated values of $\rho_{\text {step }}$ to the observed qualitative anisotropy in graphene's resistance depicted in Fig. 3. For $\rho_{\text {step }}=203 \Omega \mu \mathrm{m}, n_{\text {edges }} \sim 81$ over the distance of $600 \mu \mathrm{m}$, and sample dimensions $10 \mathrm{~mm} \times 10 \mathrm{~mm}$, the total additional resistance introduced by $\mathrm{SiC}$ step edges in the direction perpendicular to the current flow equals $R_{\text {step }}$ $=\rho_{\text {step }} \cdot N_{\text {edges }} / W_{\text {edges }}=203 \Omega \mu \mathrm{m} \cdot(10 \mathrm{~mm} \cdot 81 / 600 \mu \mathrm{m}) / 10 \mathrm{~mm}$ $=27.4 \Omega$, where $N_{\text {edges }}$ and $W_{\text {edges }}$, are the total number and width of step edges, respectively. In case of the second sample, $\rho_{\text {step }}=192 \Omega \mu \mathrm{m}, n_{\text {edges }} \sim 130, R_{\text {step }}=41.6 \Omega$. These additional step-edge-induced resistances constitute $\sim 18 \%$ and $\sim 28 \%$ of average $R_{\mathrm{S}}$ of these samples $(\sim 150 \Omega)$ measured in van der Pauw geometry. Such a contribution is less severe than it was predicted by Fig. 3, where it is suggested that over the area of $10 \mathrm{~mm} \times 10 \mathrm{~mm}$ sample, the terraces introduce around $100 \%$ the average $R_{\mathrm{S}}$. The authors believe that the overestimated anisotropy induced from van der Pauw characterization has its origin in the specificity of this technique itself. During the measurement, the current path spans only a fraction of the substrate's surface. It is narrower than the sample's width and its highest density is localized near the sample's edge between the two current contacts and thus it experiences relatively overestimated step-edgeinduced resistance.

These derived resistivities $\rho_{\text {step }}$ are higher than those discussed in Ref. 51, where for monolayer graphene, the following values were obtained: $\sim 7 \Omega \mu \mathrm{m}$ for step height $h_{S}=0.5 \mathrm{~nm}, \sim 15 \Omega \mu \mathrm{m}$ for $h_{S}=1.0 \mathrm{~nm}$, and $\sim 25 \Omega \mu \mathrm{m}$ for $h_{S}=0.5 \mathrm{~nm}$. Nearly identical resistivities for monolayer graphene were reported in Ref. 54. However, they imply atomic steps rather than macro steps that originate from step bunching. In this report, the calculated values of $\rho_{\text {step }}$, i.e., $203 \Omega \mu \mathrm{m}$ and $192 \Omega \mu \mathrm{m}$ correspond to step heights of $\sim 7.4 \mathrm{~nm}$ and $\sim 10.0 \mathrm{~nm}$. These numbers come as an average step height measured with an atomic force microscope over a distance of $2 \mathrm{~mm}$ and are found to be symptomatic for the step bunching phenomenon. Typical values of step heights and terrace widths witnessed after the growth-preceding in-situ hydrogen etching of $\mathrm{SiC}$ surface are depicted in Fig. 5.

In Ref. 54, it was found that for monolayer graphene, the mechanical deformation of graphene sheet cannot account for the observed step edge resistivity and it is rather the abrupt variation in potential and doping due to the detachment of graphene from the substrate as it passes over a step that introduces the additional scattering mechanism. To support this reasoning, we investigated a monolayer CVD graphene transferred from copper onto the $4 \mathrm{H}-\mathrm{SiC}$ substrate using the PMMA-mediated technique. To assure that the transferred graphene reproduces $\mathrm{SiC}$ surface morphology and the step edge curvature, we analyzed its bending over an

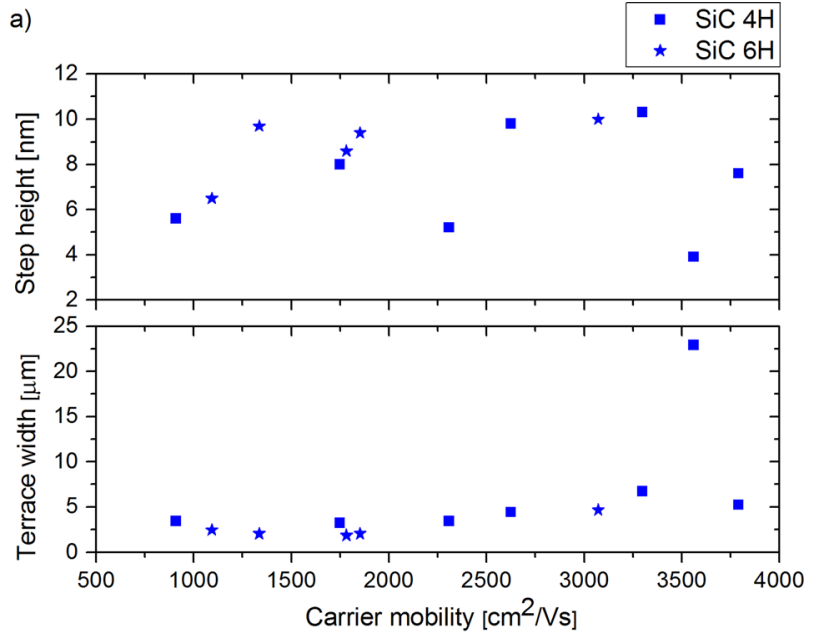

b)

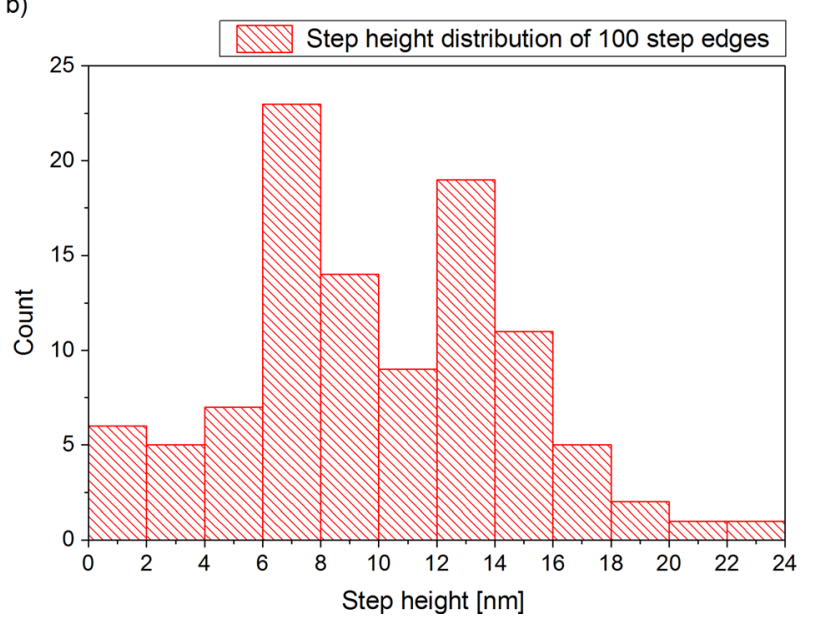

FIG. 5. (a) Typically witnessed average step heights and average terrace widths resulting from the step bunching phenomenon during the growthpreceding in-situ hydrogen etching of SiC surface (the reported values refer to $124 \mathrm{H}$ and $6 \mathrm{H}$ on-axis $\mathrm{SiC}$ samples and are averaged over a distance of $2 \mathrm{~mm}$ ). (b) Exemplary step height distribution of the consecutive $100 \mathrm{SiC}$ step edges.

etched pit dislocation. It has been confirmed using SEM imaging that graphene precisely imitates the substrate's texture. The nine rotated Hall bars were characterized accordingly to the procedure adopted for the two hydrogen intercalated $4 \mathrm{H}-\mathrm{SiC}$ samples. No recognizable pattern in resistance anisotropy was detected (Fig. 6(c)). Unlike in CVD QFS-bilayer graphene grown on $\mathrm{SiC}$, where for $\alpha_{1}<\alpha_{2}$, $R_{13}>R_{24}$, and $R_{13}<R_{24}$ when $\alpha_{1}>\alpha_{2}$, here the measured $\Delta R$ took random, both positive and negative values in the range of approximately $\pm 600 \Omega$. Bearing in mind the measured $R_{i j}$ resistance between the opposite contact pads of approximately $3.8 \mathrm{k} \Omega-8.2 \mathrm{k} \Omega$, we state that $\Delta R$ is relatively weaker $\left(<12 \%\right.$ of $\left.R_{i j}\right)$ than it was witnessed for epitaxial QFSbilayer graphene ( $\Delta R$ up to $38 \%$ of $R_{i j}, R_{i j}$ in the range of $370 \Omega-710 \Omega$ ). Thus, taking into account the lower values of $\Delta R$ relative to $R_{i j}$ and their random nature, we attribute the data scatter to inhomogeneities in local transport properties of the transferred graphene. This observation provides further proof that the step edge resistivity cannot originate from graphene's mechanical deformation. This conclusion suggests that the charge carriers are not subject to scattering 


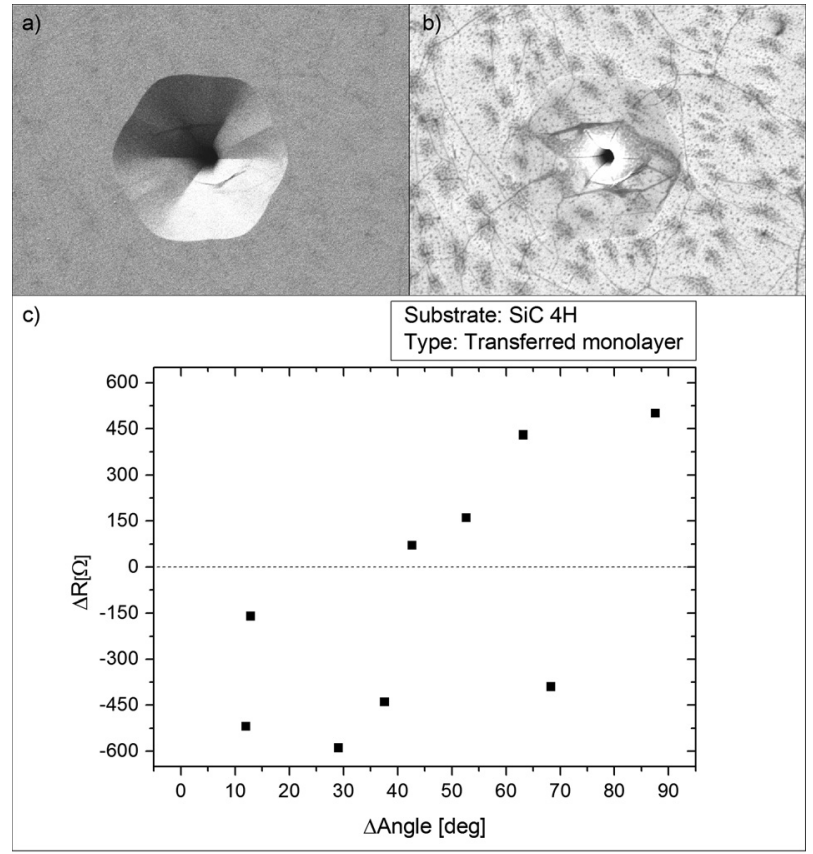

FIG. 6. (a) Scanning electron microscopy image of an etched pit dislocation ( $4 \mu \mathrm{m}$ in diameter) before and (b) after graphene transfer. Graphene's ability to reproduce surface morphology is proven by the smooth coverage of the pit slopes. (c) Differential resistance $\Delta R$ measured in nine pairs of mutually perpendicular graphene channels $(200 \mu \mathrm{m} \times 600 \mu \mathrm{m})$ as a function of the differential angle $\Delta \alpha$ between the terraces orientation in each pair of the mutually perpendicular channels in monolayer CVD copper-grown graphene transferred onto $4 \mathrm{H}-\mathrm{SiC}(0001)$ substrate.

over the substrates steps and consequently the step edge area does not influence their mobility. Bearing in mind that the resistance is a product of both carrier mobility $\mu$ and concentration $n\left(R^{-1}=e n \mu\right)$, we conclude that the derived $\rho_{\text {step }}$ may originate from a decreased carrier population in the step area.

The possible carrier population depletion is expected to manifest itself in Raman spectroscopy imaging of the terrace and step edge area. ${ }^{62}$ Micro-Raman $24 \mu \mathrm{m} \times 16 \mu \mathrm{m}$ maps of a hydrogen intercalated QFS-bilayer graphene within the terrace and step edge area, performed in a backscattering geometry using an inVia Renishaw microscope powered by a 532 nm CW Nd-YAG laser, are depicted in Figs. 7 and 8.

The number of graphene layers is verified in two ways. First, the FWHM of the 2D band within the terrace area is $\sim 60 \mathrm{~cm}^{-1}$ and it reaches $\sim 70 \mathrm{~cm}^{-1}$ on the step edges (Fig. $8(\mathrm{~b})$ ). It has been shown that exfoliated bilayer graphene exhibits a 2D FWHM of $\sim 50 \mathrm{~cm}^{-1}$ (Ref. 63) and other reports yield a range of $41-60 \mathrm{~cm}^{-1} \cdot{ }^{64-68}$ Therefore, we reason that the terraces are covered with bilayer graphene and that there is an additional graphene layer in the step edge area. Second, bilayer graphene is expected to yield an asymmetric 2D band that is only reproduced by a sum of four Lorentzians, whereas the 2D band of a trilayer graphene is symmetrical and may by approximated by a single Voigt curve. Following the procedure adopted in Ref. 69, we analyze the overall quality of fitting the measured 2D band with a set of four Lorentzian curves and with a single Voigt function. An exemplary Raman spectrum within the terrace and
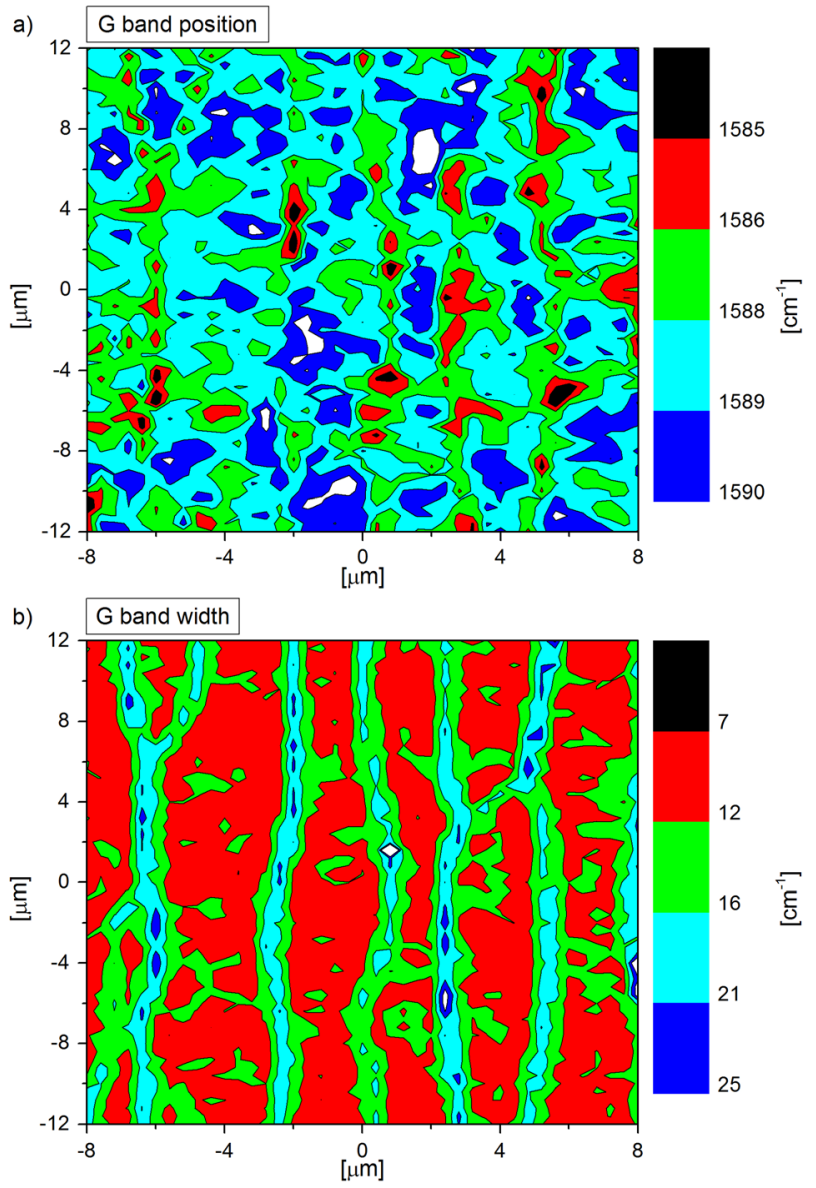

FIG. 7. Micro-Raman $24 \mu \mathrm{m} \times 16 \mu \mathrm{m}$ map of QFS-bilayer epitaxial CVD graphene on SiC. (a) G band position and (b) G band width.

step edge area is presented in Fig. 9. The comparison of the chi-squared value of the fitting correctness of the $2 \mathrm{D}$ band with the above mentioned functions (Figs. 10(a) and10(b)) proves that the terrace area is better approximated with a four-fold Lorentzian whereas the step edge area by a single Voigt, which suggests that the step edges are decorated with an additional (third) graphene layer.

In Ref. 70, it was clearly presented that under biaxial strain conditions, when the position of the $\mathrm{G}$ band in bilayer graphene is rising, the position of the $2 \mathrm{D}$ band is also increased. This is in contrast to the step edges, where an observable blue-shift of the 2D band is followed by a redshift of the G band (Figs. 8(a) and 7(a)). We attribute the mechanism responsible for the blue-shift of the $2 \mathrm{D}$ band predominantly to strain induced by the step edges. Taking into account the fact that a deviation from a consistent shift of the $\mathrm{G}$ band and 2D band positions is mostly an evidence of carrier concentration changes and that in our experiment the red-shift of the $\mathrm{G}$ band position (Fig. 7(a)) is followed by a sharp increase in its width (Fig. 7(b)), ${ }^{71}$ we reason from the Raman results that carrier concentration is lowered at the step edges as compared with the terraces.

It is assumed in Ref. 54 that along the detachment length $l_{d} \approx 1.2 h_{S}$, where $h_{S}$ is the step height, graphene is fully depleted of carriers. As a result, the step edge resistivity is expected to scale linearly with the step height. In our 

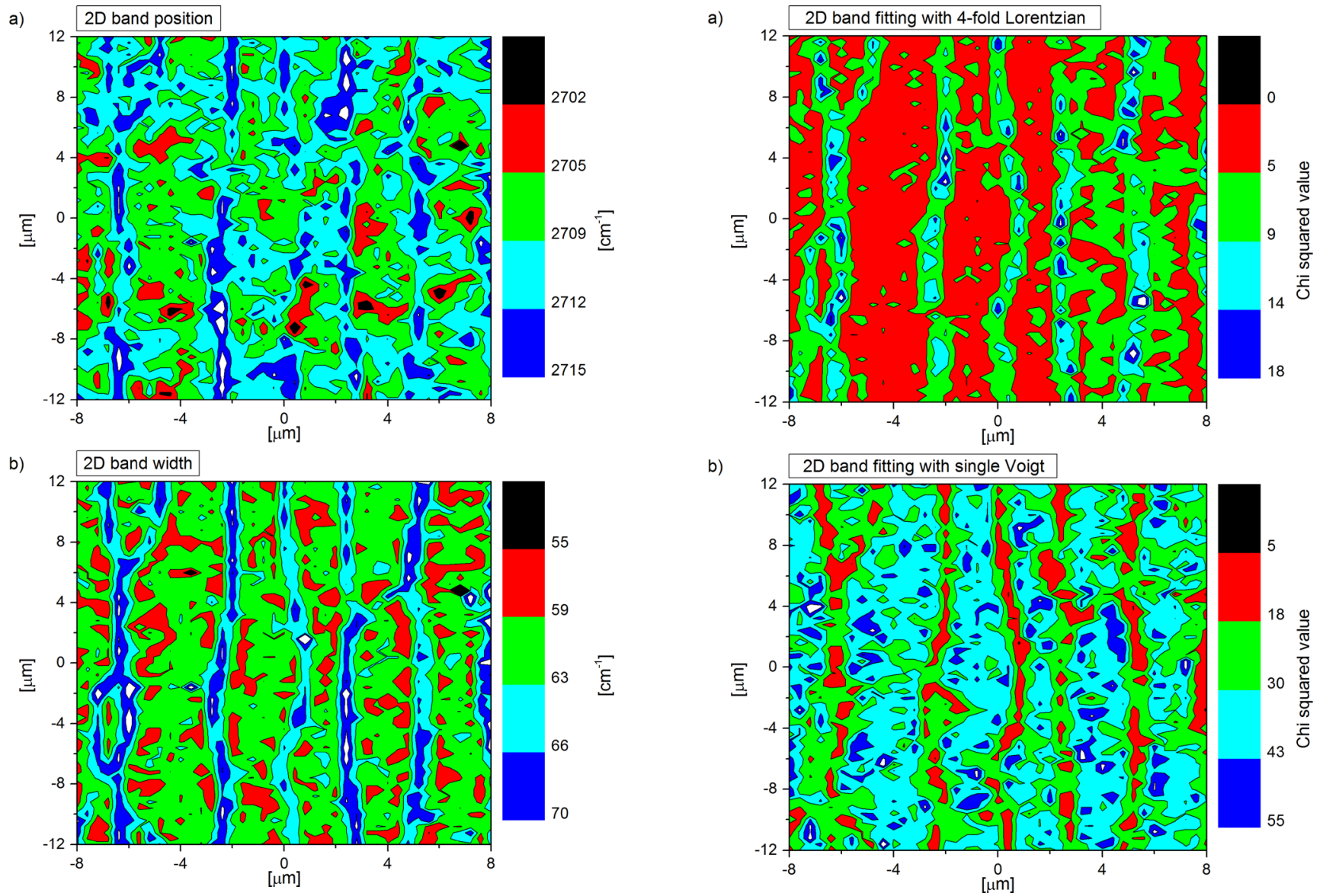

FIG. 8. Micro-Raman $24 \mu \mathrm{m} \times 16 \mu \mathrm{m}$ map of QFS-bilayer epitaxial CVD graphene on $\mathrm{SiC}$. (a) 2D band position and (b) 2D band width.

experiment, the linear scaling is only supported for the orderly oriented terraces (Fig. 11). It has been observed that the meandering geometry promoted a $\sim 50 \%$ higher step edge resistivity than it was expected for given $h_{S}$. On the basis of the fine agreement of our result derived for the orderly

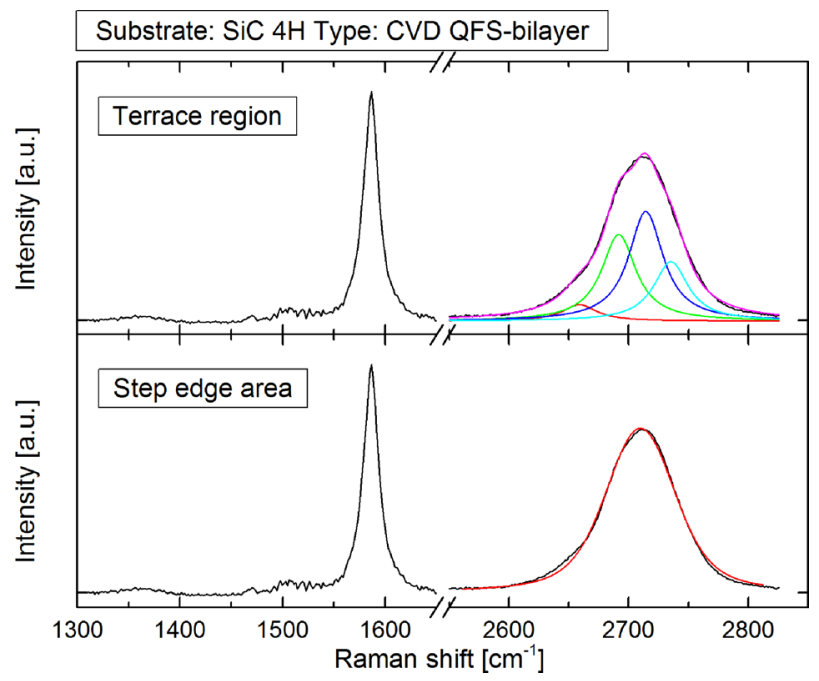

FIG. 9. Exemplary Raman spectrum within the terrace and step edge area. (a) In the terrace region, the $2 \mathrm{D}$ band is better approximated by a sum of four Lorentzian curves. (b) In the step edge area, the 2D band is better approximated by a single Voigt function.

FIG. 10. Micro-Raman $24 \mu \mathrm{m} \times 16 \mu \mathrm{m}$ map of QFS-bilayer epitaxial CVD graphene on SiC. (a) Residual error of 2D band fit with a 4-fold Lorentzian. (b) Residual error of 2D band fit with a single Voigt function.

oriented terraces with the linear approximation of the reported data, we conclude that the adopted explanation for $\rho_{\text {step }}$ origin is reliable. Yet, the overestimated step edge

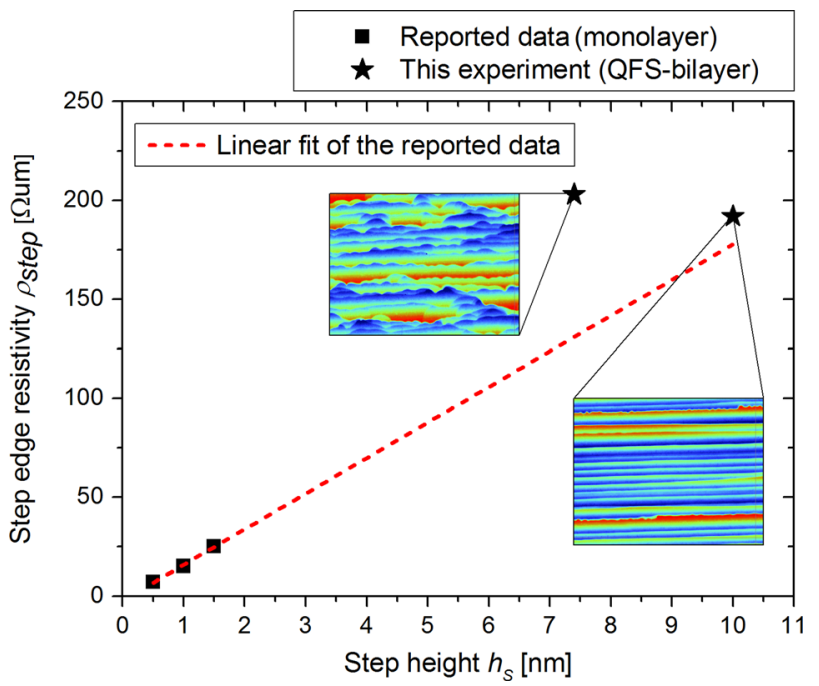

FIG. 11. Comparison of the reported values ${ }^{51,54}$ of the step edge resistivity related to $\mathrm{SiC}$ atomic steps with our results for $\rho_{\text {step }}$ indicative of the step bunching occurring during the growth-preceding in-situ hydrogen etching of the $\mathrm{SiC}$ surface. Inset imaging obtained with a Bruker ContourGT-I 3D optical microscope depicts surface morphology of the investigated samples (meandering and orderly oriented terraces). 
resistance of the winding terraces may suggest that graphene's resistance is further augmented by local morphology. Such contribution could be a consequence of residual Si atoms aggregated in the step edge area ${ }^{55}$ or growth disorder near the step edges leading to deterioration of graphene's quality and promoting short-range scattering. ${ }^{72}$

\section{CONCLUSIONS}

We showed the qualitative influence of SiC step edge orientation on the resistance of hydrogen intercalated QFS-bilayer graphene grown on $4 \mathrm{H}-\mathrm{SiC}(0001)$ and $6 \mathrm{H}-\mathrm{SiC}(0001)$ $(10 \mathrm{~mm} \times 10 \mathrm{~mm})$ by chemical vapor deposition. The statistical overview of 200 samples marks a distinctive relation between terrace orientation and the excess resistance. Similar results are observed for $4 \mathrm{H}$ and $6 \mathrm{H}$ polytypes. A further detailed analysis yields exact values of step edge resistivity in QFS-bilayer graphene on $4 \mathrm{H}-\mathrm{SiC}$ substrate $\rho_{\text {step }}=203 \Omega \mu \mathrm{m}$ for $h_{S}=7.4 \mathrm{~nm}$ and $\rho_{\text {step }}=192 \Omega \mu \mathrm{m}$ for $h_{S}=10.0 \mathrm{~nm}$. In the case of orderly oriented terraces, our result is in agreement with the previously reported values for the step-height-scaling resistivity in monolayer graphene. It was observed that the meandering geometry of the terraces promoted a $\sim 50 \%$ higher step edge resistivity than it was expected for given $h_{S}$. No clear pattern was observed in the resistance anisotropy of the copper-grown graphene transferred onto a $4 \mathrm{H}-\mathrm{SiC}$ substrate that would indicate the deformation-induced step edge resistivity. The results suggest that the adopted explanation for $\rho_{\text {step }}$ origin, graphene's depletion of carriers over the detachment length, is reliable but this effect may be further augmented by growth disorder near the step edges and consequent short-range scattering. The authors believe that the typical macro step height $(\sim 10 \mathrm{~nm})$ arising from the step bunching promoted by the in situ hydrogen etching of the $\mathrm{SiC}$ substrate gives rise to a non-negligible carrier transport impediment and should be considered in the design of the micro-scale graphene-based devices. In our analysis, we focused on large-scale, statistical parameters that will foster the reproducibility and standardization of the academicscale technology and provide basis for the scalable, industrial, high-yield graphene production for electronics and sensor technologies.

\section{ACKNOWLEDGMENTS}

The authors thank Professor Jacek Baranowski for many useful discussions. The research leading to these results has received funding from the European Union Seventh Framework Programme under Grant Agreement No. 604391 Graphene Flagship. This work was also partially supported by the National Centre for Research and Development under the GRAF-TECH/NCBiR/01/32/2012 "GRAFMET" grant and GRAF-TECH/NCBiR/12/14/2013 "GRAFMAG" grant, the Ministry of Science and Higher Education under the Iuventus Plus 0296/IP2/2013/72 grant and the National Science Centre under the PRELUDIUM 2013/11/N/ST3/04147 grant.

${ }^{1}$ P. Avouris, "Graphene: Electronic and photonic properties and devices," Nano Lett. 10, 4285 (2010).

${ }^{2}$ Y.-M. Lin, A. Valdes-Garcia, S.-J. Han, D. B. Farmer, I. Meric, Y. Sun et al., "Wafer-scale graphene integrated circuit," Science 332, 1294-1297 (2011).
${ }^{3}$ L. Wang, I. Meric, P. Y. Huang, Q. Gao, Y. Gao, H. Tran, T. Taniguchi, $\mathrm{K}$. Watanabe et al., "One-dimensional electrical contact to q twodimensional material," Science 342, 614 (2013).

${ }^{4}$ A. K. Geim and K. S. Novoselov, "The rise of graphene," Nature Mater. 6, 183-191 (2007).

${ }^{5}$ S. Novoselov, A. K. Geim, S. V. Morozov, D. Jiang, Y. Zhang, S. V. Dubonos et al., "Electric field effect in atomically thin carbon films," Science 306, 666-669 (2004).

${ }^{6}$ Y. Zhang, J. W. Tan, H. L. Stormer, and P. Kim, "Experimental observation of the quantum Hall effect and Berry's phase in graphene," Nature 438, 201-204 (2005).

${ }^{7}$ V. E. Dorgan, A. Behnam, H. J. Conley, K. I. Bolotin, and E. Pop, "Highfield electrical and thermal transport in suspended graphene," Nano Lett. 13, 4581-4586 (2013).

${ }^{8}$ K. I. Bolotin, K. J. Sikes, Z. Jiang, M. Klima, G. Fudenberg, J. Hone, P. Kim, and H. L. Stormer, "Ultrahigh electron mobility in suspended graphene," Solid State Commun. 146, 351-355 (2008).

${ }^{9}$ X. Du, I. Skachko, A. Barker, and E. Y. Andrei, "Approaching the ballistic transport in suspended graphene," Nat. Nanotechnol. 3, 491-495 (2008).

${ }^{10} \mathrm{~T}$. Ando, "Screening effect and impurity scattering in monolayer graphene," J. Phys. Soc. Jpn. 75, 074716 (2006).

${ }^{11}$ K. Nomura and A. H. MacDonald, "Quantum transport of massless Dirac fermions," Phys. Rev. Lett. 98, 076602 (2007).

${ }^{12}$ V. V. Cheianov and V. I. Fal'ko, "Friedel oscillations, impurity scattering, and temperature dependence of resistivity in graphene," Phys. Rev. Lett. 97, 226801 (2006).

${ }^{13}$ E. H. Hwang, S. Adam, and S. Das Sarma, "Carrier transport in twodimensional graphene layers,” Phys. Rev. Lett. 98, 186806 (2007).

${ }^{14}$ S. Adam, E. H. Hwang, V. M. Galitski, and S. Das Sarma, "A selfconsistent theory for graphene transport," Proc. Natl. Acad. Sci. U.S.A. 104, 18392 (2007).

${ }^{15}$ D. S. Novikov, "Numbers of donors and acceptors from transport measurements in graphene," Appl. Phys. Lett. 91, 102102 (2007).

${ }^{16} \mathrm{M}$. Trushin and J. Schliemann, "The minimum electrical and thermal conductivity of graphene: Quasiclassical approach," Phys. Rev. Lett. 99, 216602 (2007).

${ }^{17}$ X.-Z. Yan, Y. Romiah, and C. S. Ting, "Electric transport theory of Dirac fermions," Phys. Rev. B 77, 125409 (2008).

${ }^{18}$ J.-H. Chen, C. Jang, S. Adam, M. S. Fuhrer, E. D. Williams, and M. Ishigami, "Charged-impurity scattering in graphene," Nat. Phys. 4, 377-381 (2008).

${ }^{19}$ W. Zhu, V. Perebeinos, M. Freitag, and P. Avouris, "Carrier scattering, mobilities, and electrostatic potential in monolayer, bilayer, and trilayer graphene," Phys. Rev. B 80, 235402 (2009).

${ }^{20}$ D. B. Farmer, V. Perebeinos, Y.-M. Lin, C. Dimitrakopoulos, and P. Avouris, "Charge trapping and scattering in epitaxial graphene," Phys. Rev. B 84, 205417 (2011).

${ }^{21}$ Y.-W. Tan, Y. Zhang, K. Bolotin, Y. Zhao, S. Adam, E. H. Hwang, S. Das Sarma, H. L. Stormer, and P. Kim, "Measurement of scattering rate and minimum conductivity in graphene," Phys. Rev. Lett. 99, 246803 (2007).

${ }^{22}$ C. Jang, S. Adam, J.-H. Chen, E. D. Williams, S. Das Sarma, and M. S. Fuhrer, "Tuning the effective fine structure constant in graphene: Opposing effects of dielectric screening on short- and long-range potential scattering," Phys. Rev. Lett. 101, 146805 (2008).

${ }^{23}$ D. S. Novikov, "Elastic scattering theory and transport in graphene," Phys. Rev. B 76, 245435 (2007)

${ }^{24}$ S. Adam and S. Das Sarma, "Boltzmann transport and residual conductivity in bilayer graphene,” Phys. Rev. B 77, 115436 (2008).

${ }^{25}$ S. Das Sarma, E. H. Hwang, and E. Rossi, "Theory of carrier transport in bilayer graphene," Phys. Rev. B 81, 161407 (2010).

${ }^{26}$ J. W. Klos and I. V. Zozoulenko, "Effect of short- and long-range scattering on the conductivity of graphene: Boltzmann approach vs tight-binding calculations,” Phys. Rev. B 82, 081414 (2010).

${ }^{27}$ S. Fratini and F. Guinea, "Substrate-limited electron dynamics in graphene," Phys. Rev. B 77, 195415 (2008).

${ }^{28}$ V. W. Brar, Y. Zhang, Y. Yayon, T. Ohta, J. L. McChesney, A. Bostwick, E. Rotenberg, K. Horn, and M. F. Crommie, "Scanning tunneling spectroscopy of inhomogeneous electronic structure in monolayer and bilayer graphene on SiC,” Appl. Phys. Lett. 91, 122102 (2007).

${ }^{29}$ Y. Zhang, V. W. Brar, C. Girit, A. Zettl, and M. F. Crommie, "Origin of spatial charge inhomogeneity in graphene," Nat. Phys. Lett. 5(10), 722-726 (2009).

${ }^{30}$ A. Castellanos-Gomez, R. H. M. Smit, N. Agrait, and G. Rubio-Bollinger, "Spatially resolved electronic inhomogeneities of graphene due to subsurface charges," Carbon 50, 932-938 (2012). 
${ }^{31}$ K. Ziegler, "Robust transport properties in graphene," Phys. Rev. Lett. 97, 266802 (2006).

${ }^{32}$ N. M. R. Peres, F. Guinea, and A. H. Castro Neto, "Electronic properties of disordered two-dimensional carbon," Phys. Rev. B 73, 125411 (2006).

${ }^{33}$ E. A. Kim and A. H. Castro Neto, "Graphene as an electronic membrane," EPL 84, 57007 (2008).

${ }^{34}$ M. I. Katsnelson and A. K. Geim, "Electron scattering on microscopic corrugations in graphene," Philos. Trans. R. Soc. A 366, 195-204 (2008).

${ }^{35}$ H. Kuramochi, S. Odaka, K. Morita, S. Tanaka, H. Miyazaki, M. V. Lee, S.-L. Li, H. Hiura, and K. Tsukagoshi, "Role of atomic terraces and steps in the electron transport properties of epitaxial graphene grown on $\mathrm{SiC}$,". AIP Adv. 2, 012115 (2012).

${ }^{36} \mathrm{~A}$. Fasolino, J. H. Los, and M. I. Katsnelson, "Intrinsic ripples in graphene," Nature Mater. 6, 858-861 (2007).

${ }^{37}$ F. de Juan, A. Cortijo, and M. A. H. Vozmediano, "Charge inhomogeneities due to smooth ripples in graphene sheets," Phys. Rev. B 76, 165409 (2007).

${ }^{38}$ I. F. Herbut, V. Juricic, and O. Vafek, "Coulomb interaction, ripples, and the minimal conductivity of graphene," Phys. Rev. Lett. 100, 046403 (2008).

${ }^{39}$ J.-H. Chen, C. Jang, S. Xiao, M. Ishigami, and M. S. Fuhrer, "Intrinsic and extrinsic performance limits of graphene devices on $\mathrm{SiO}_{2}$," Nat. Nanotechnol. 3, 206-209 (2008).

${ }^{40}$ E. H. Hwang and S. Das Sarma, "Acoustic phonon scattering limited carrier mobility in two-dimensional extrinsic graphene," Phys. Rev. B 77, 115449 (2008).

${ }^{41}$ S. V. Morozov, K. S. Novoselov, M. I. Katsnelson, F. Schedin, D. C. Elias, J. A. Jaszczak, and A. K. Geim, "Giant intrinsic carrier mobilities in graphene and its bilayer," Phys. Rev. Lett. 100, 016602 (2008).

${ }^{42}$ Y.-M. Lin, C. Dimitrakopoulos, K. A. Jenkins, D. B. Farmer, H.-Y. Chiu, A. Grill, and $\mathrm{Ph}$. Avouris, "100-GHz transistors from wafer-scale epitaxial graphene," Science 327, 662 (2010).

${ }^{43}$ J. Hass, R. Feng, T. Li, X. Li, Z. Zong, W. A. de Heer, P. N. First, and E. H. Conrad, "Highly ordered graphene for two dimensional electronics," Appl. Phys. Lett. 89, 143106 (2006).

${ }^{44}$ W. A. de Heer, C. Berger, M. Ruan, M. Sprinkle, X. Li, Y. Hu, B. Zhang, J. Hankinson, and E. Conrad, "Large area and structured epitaxial graphene produced by confinement controlled sublimation of silicon carbide," Proc. Natl. Acad. Sci. U.S.A. 108, 16900-16905 (2011).

${ }^{45}$ C. Berger, J.-Y. Veuillen, L. Magaud, P. Mallet, V. Olevano, M. Orlita, and P. Plochocka, "Electronic properties of epitaxial graphene," Int. J. Nanotechnol. 7, 383-402 (2010).

${ }^{46}$ W. Strupinski, K. Grodecki, A. Wysmolek, R. Stepniewski, T. Szkopek, and P. E. Gaskell, "Graphene epitaxy by chemical vapor deposition on SiC," Nano Lett. 11(4), 1786-1791 (2011).

${ }^{47}$ K. V. Emtsev, A. Bostwick, K. Horn, J. Jobst, G. L. Kellogg, L. Ley, J. L. McChesney, T. Ohta, S. A. Reshanov, J. Röhrl, E. Rotenberg, A. K. Schmid, D. Waldmann, H. B. Weber, and T. Seyller, "Towards wafer-size graphene layers by atmospheric pressure graphitization of silicon carbide," Nature Mater. 8, 203-207 (2009).

${ }^{48}$ M. J. Hollander, A. Agrawal, M. S. Bresnehan, M. LaBella, K. A. Trumbull, R. Cavalero, D. W. Snyder, S. Datta, and J. A. Robinson, "Heterogeneous integration of hexagonal boron nitride on bilayer quasifree-standing epitaxial graphene and its impact on electrical transport properties," Phys. Status Solidi A 210, 1062-1070 (2013).

${ }^{49}$ Y. Guo, L. W. Guo, J. Huang, R. Yang, Y. P. Jia, J. J. Lin, W. Lu, Z. L. $\mathrm{Li}$, and $\mathrm{X}$. L. Chen, "The correlation of epitaxial graphene properties and morphology of SiC (0001)," J. Appl. Phys. 115, 043527 (2014).

${ }^{50}$ C. Dimitrakopoulos, A. Grill, T. McArdle, Z. Liu, R. Wisnieff, and D. A. Antoniadis, "Effect of $\mathrm{SiC}$ wafer miscut angle on the morphology and Hall mobility of epitaxially grown graphene," Appl. Phys. Lett. 98, 222105 (2011).

${ }^{51}$ S.-H. Ji, J. B. Hannon, R. M. Tromp, V. Perebeinos, J. Tersoff, and F. M. Ross, "Atomic-scale transport in epitaxial graphene," Nature Mater. 11, 114-119 (2011).

${ }^{52}$ S. E. Bryan, Y. Yang, and R. Murali, "Conductance of epitaxial graphene nanoribbons: Influence of size effects and substrate morphology," J. Phys. Chem. C 115, 10230-10235 (2011).
${ }^{53}$ C. H. Lui, L. Liu, K. F. Mak, G. W. Flynn, and T. F. Heinz, "Ultraflat graphene," Nature 462, 339-341 (2009).

${ }^{54}$ T. Low, V. Perebeinos, J. Tersoff, and Ph. Avouris, "Deformation and scattering in graphene over substrate steps," Phys. Rev. Lett. 108, 096601 (2012).

${ }^{55}$ M. K. Yakes, D. Gunlycke, J. L. Tedesco, P. M. Campbell, R. L. MyersWard, C. R. Eddy, Jr., K. Gaskill, P. E. Sheehan, and A. R. Laracuente, "Conductance anisotropy in epitaxial graphene sheets generated by substrate interactions," Nano Lett. 10, 1559-1562 (2010).

${ }^{56}$ N. Ray, S. Shallcross, S. Hensel, and O. Pankratov, "Buffer layer limited conductivity in epitaxial graphene on the Si face of SiC," Phys. Rev. B 86, 125426 (2012).

${ }^{57}$ C. Riedl, U. Starke, J. Bernhardt, M. Franke, and K. Heinz, "Structural properties of the graphene-SiC $(0001)$ interface as a key for the preparation of homogeneous large-terrace graphene surfaces," Phys. Rev. B 76, 245406 (2007).

${ }^{58}$ K. V. Emtsev, F. Speck, Th. Seyller, L. Ley, and J. D. Riley, "Interaction, growth, and ordering of epitaxial graphene on $\mathrm{SiC}\{0001\}$ surfaces: A comparative photoelectron spectroscopy study: A comparative photoelectron spectroscope study," Phys. Rev. B 77, 155303 (2008).

${ }^{59}$ C. Riedl, C. Coletti, T. Iwasaki, A. A. Zakharov, and U. Starke, "Quasifree-standing epitaxial graphene on $\mathrm{SiC}$ obtained by hydrogen intercalation," Phys. Rev. Lett. 103, 246804 (2009).

${ }^{60}$ L. J. van der Pauw, "A method for measuring the resistivity and Hall coefficient on lamellae arbitarty shape," Philips Res. Rep. 13, 1-9 (1958).

${ }^{61}$ T. Ciuk, I. Pasternak, A. Krajewska, J. Sobieski, P. Caban, J. Szmidt, and W. Strupinski, "Properties of chemical vapor deposition graphene transferred by high-speed electrochemical delamination," J. Phys. Chem. C 117, 20833-20837 (2013)

${ }^{62}$ K. Grodecki, R. Bozek, W. Strupinski, A. Wysmolek, R. Stepniewski, and J. M. Baranowski, "Micro-Raman spectroscopy of graphene grown on stepped 4H-SiC (0001) surface,” Appl. Phys. Lett. 100, 261604 (2012).

${ }^{63}$ A. C. Ferrari, J. C. Meyer, V. Scardaci, C. Casiraghi, M. Lazzeri, F. Mauri, S. Piscanec, D. Jiang, K. S. Novoselov, S. Roth, and A. K. Geim, "Raman spectrum of graphene and graphene layers," Phys. Rev. Lett. 97(18), 187401 (2006).

${ }^{64}$ J. A. Robinson, M. Wetherington, J. L. Tedesco, P. M. Campbell, X. Weng, J. Stitt, M. A. Fanton, E. Frantz, D. Snyder, B. L. Vanmil, G. G. Jernigan, R. L. Myers-Ward, C. R. Eddy, Jr., and D. K. Gaskill, "Correlating Raman spectral signatures with carrier mobility in epitaxial graphene: a guide to achieving high mobility on the wafer scale," Nano Lett. 9(8), 2873-2876 (2009).

${ }^{65}$ J. Röhrl, M. Hundhausen, K. V. Emtsev, Th. Seyller, R. Graupner, and L. Ley, "Raman spectra of epitaxial graphene on SiC(0001)," Appl. Phys. Lett. 92(20), 201918 (2008).

${ }^{66}$ P. Lespade, A. Marchand, M. Couzi, and F. Cruege, Carbon 22, 375 (1984).

${ }^{67}$ A. Ferrari and J. Robertson, "Interpretation of Raman spectra of disordered and amorphous carbon," Phys. Rev. B 61, 14095 (2000).

${ }^{68}$ L. M. Malard, M. A. Pimenta, G. Dresselhaus, and M. S. Dresselhaus, "Raman spectroscope in graphene," Phys. Rep. 473, 51-87 (2009).

${ }^{69}$ L. O. Nyakiti, R. L. Myers-Ward, V. D. Wheeler, E. A. Imhoff, F. J. Bezares, H. Chun, J. D. Caldwell, A. L. Friedman, B. R. Matis, J. W. Baldwin, P. M. Campbell, J. C. Culbertson, C. R. Eddy, Jr., G. G. Jernigan, and D. K. Gaskill, "Bilayer graphene grown on 4H-SiC (0001) step-free mesas," Nano Lett. 12, 1749-1756 (2012).

${ }^{70}$ J. Zabel, R. R. Nair, A. Ott, T. Georgiou, A. K. Geim, K. S. Novoselov, and C. Casiraghi, "Raman spectroscopy of graphene and bilayer under biaxial strain: Bubbles and balloons," Nano Lett. 12(2), 617-621 (2012).

${ }^{71}$ J. Yan, T. Villarson, E. A. Henriksen, Ph. Kim, and Aaron Pinczuk, "Optical phonon mixing in bilayer graphene with a broken inversion symmetry,” Phys. Rev. B 80, 241417 (2009).

${ }^{72}$ W. Strupinski, K. Grodecki, P. Caban, P. Ciepielewski, and J. M. Baranowski, "Formation mechanism of graphene buffer layer on $\mathrm{SiC}(0001)$," Carbon (to be published). 